\title{
Stem cells in regenerative medicine - from laboratory to clinical application - the eye
}

\author{
ANNA M. DAZBROWSKA ${ }^{1}$, PIOTR SKOPIŃSKI ${ }^{1,2}$ \\ ${ }^{1}$ Department of Ophthalmology, Second Faculty of Medicine, Warsaw Medical University, Poland \\ ${ }^{2}$ Department of Histology and Embriology, Biostructure Center, Warsaw Medical University, Poland
}

\begin{abstract}
Stem cells are currently one of the most researched and explored subject in science. They consstitue a very promising part of regenerative medicine and have many potential clinical applications. Harnessing their ability to replicate and differentiate into many cell types can enable successful treatment of diseases that were incurable until now. There are numerous types of stem cells (e.g. ESCs, FSCs, ASCs, iPSCs) and many different methods of deriving and cultivating them in order to obtain viable material. The eye is one of the most interesting targets for stem cell therapies. In this article we summarise different aspects of stem cells, discussing their characteristics, sources and methods of culture. We also demonstrate the most recent clinical applications in ophthalmology based on an extensive current literature review. Tissue engineering techniques developed for corneal limbal stem cell deficiency, age-related macular degeneration (AMD) and glaucoma are among those presented. Both laboratory and clinical aspects of stem cells are discussed.
\end{abstract}

Key words: stem cells, tissue engineering, regenerative medicine, eye, biomarkers, AMD, glaucoma, limbal stem cell deficiency, ophthalmology.

(Cent Eur J Immunol 2017; 42 (2):173-180)

\section{Introduction}

Stem cells present multiple potential applications in regenerative medicine and are the subject of intense research [1]. The eye is a fascinating and promising target for stem cell therapies because it is a relatively immunologically privileged and surgically accessible self-contained system. Many ocular diseases such as corneal limbal stem cell deficiency, age-related macular degeneration (AMD), glaucoma, or retinal dystrophies could be treated with the use of tissue engineering.

In this article, the authors summarise different aspects of stem cell therapy, discussing the nature of stem cells, their sources and culture, and presenting the most recent clinical applications in ophthalmology as well as potential uses in future.

\section{Classification}

Stem cells are defined by their ability to regenerate multiple differentiated cell types, while retaining the capacity to self-replicate (Simonovitch et al. 1963). Those found in vivo have different origin and can be divided into 3 broad categories accordingly: embryonic (ESCs), foetal (FSCs) and adult stem cells (ASCs, among them mesenchymal stem cells - MSCs). Embryonic cells are plurip- otent, derived from the inner cell mass of the blastocyst, a stage of the pre-implantation embryo, 5-6 days post-fertilization [2]. They generate the organism, whereas the surrounding trophoblast cells contribute to the placental chorion. FSCs are multipotent cells located in the foetal tissues and embryonic annexes [3]. They have been subdivided into haematopoietic (blood, liver, bone marrow), mesenchymal (blood, liver, bone marrow, lung, kidney and pancreas), endothelial (bone marrow, placenta), epithelial (liver, pancreas) and neural ones (brain, spinal cord) [4]. Among FSCs the greatest potential use in regenerative medicine have stem cells found in foetal blood and in placenta because they are the easiest to harvest without harming the foetus. ASCs are multipotent tissue-resident stem cells, also termed progenitor cells, found in fully developed tissues. They reside in niches that create a special microenvironment for their replication and self-renewal.

Very important for regenerative medicine are cell's plasticity and ability to undergo the process of transdifferentiation. These two refer to the ability of some cells to give rise to cell types, formerly considered outside their normal repertoire of differentiation for the location where they are found [5]. Plasticity is the capacity of organisms or cells to alter their phenotype in response to changes in their environment [6]. Transdifferentiation is the transformation of

Correspondence: Anna M. Dąbrowska, Department of Ophthalmology, Second Faculty of Medicine, Warsaw Medical University, Poland, e-mail: anna.m.dabrowska@gmail.com Submitted: 29.10.2016; Accepted: 25.11.2016 


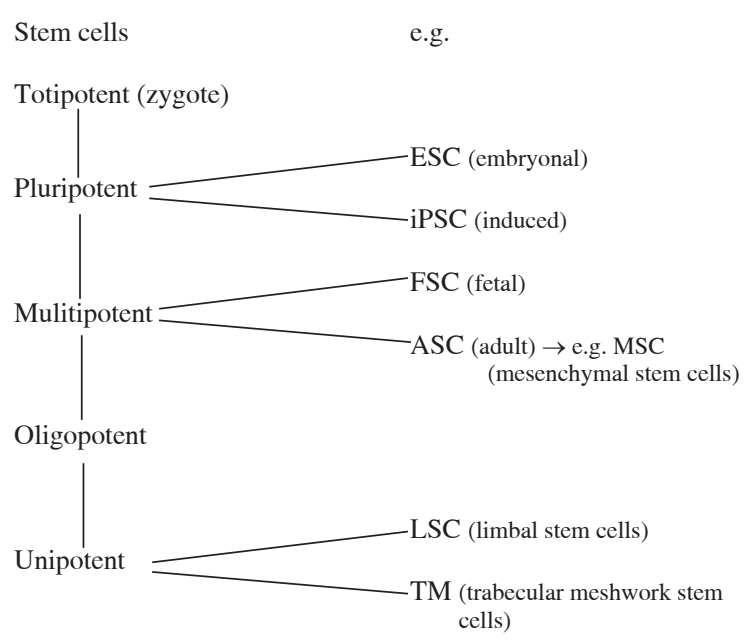

Fig. 1. Different stem cells: based on their differentiation potential stem cells can be described as totipotent, pluripotent, mulitipotent, oligopotent or unipotent [9]. Totipotent stem cells derive from an early progeny of the zygote up to the eight cell stage of the morula and have the ability to form an entire organism and the extraembryonic membranes $[10,11]$. Pluripotent cells can differentiate into tissue from all 3 germ layers (endoderm, mesoderm, and ectoderm). Multipotent stem cells may differentiate into tissue derived from a single germ layer such as mesenchymal stem cells which form adipose tissue, bone, and cartilage. Oligopotent stem cells, also called tissue-resident stem cells, can form terminally differentiated cells of a specific tissue [12]. Unipotent stem cells form a single lineage (ex. spermatogonial stem cells) [1]

a non-stem cell into a different cell type or the production of cells from a differentiated stem cell that are not related to its already established differentiation path [7].

The discovery of those processes broadened the possibilities to derive stem cells from tissues. Takahashi and Yamanaka proved in 2006 that to reprogram a differentiated cell into an embryonic-like state it is enough to introduce certain transcription factors into culture conditions [8]. Their research showed that the use of retroviral transduction enables somatic cell reprogramming into stem cells without the need of transferring their nuclear contents into oocytes or fusing them with embryonic stem cells. Cells derived by this new method are called induced pluripotent stem cells (iPSCs; Fig. 1).

Since 2006 the strategies for deriving iPSCs are constantly being improved. DNA-free and viral-free protocols have been presented using recombinant proteins, messenger RNA (mRNA) and mature microRNA (miRNA) [1315]. There has been also first attempt of in vivo reprogramming which showed that it is possible to produce totipotent iPSCs within tissues, but the technique needs major refine-
Table 1. Human embryonic stem cell (hESCs) markers [19]

\begin{tabular}{ll}
\hline hESCs markers & glycolipid antigens \\
\hline SSEA3, SSEA4 & $\begin{array}{l}\text { keratan sulfate } \\
\text { antigens }\end{array}$ \\
\hline TRA-1-60, TRA-1-81, GCTM2, GCT343 & protein antigens \\
\hline $\begin{array}{l}\text { CD9, Thy1 (CD90), tissue-nonspecific } \\
\text { alkaline phosphatase, class 1 HLA }\end{array}$ & $\begin{array}{l}\text { strongly } \\
\text { NANOG, POU5F1 (formerly known as } \\
\text { OCT4), TDGF1, DNMT3B, GABRB3, } \\
\text { GDF3 }\end{array}$ \\
\hline
\end{tabular}

Table 2. Criteria for MSCs - cell surface antigens

\begin{tabular}{ll}
\hline Express & Not express \\
\hline CD90, CD105, CD73 & CD45, CD34, CD14, CD11b, \\
& CD79 $\alpha$, CD19, HLA-DR \\
\hline
\end{tabular}

ment before it can be used in regenerative medicine as it resulted in teratomas formation so far [16].

\section{Stem cells markers}

Molecular biomarkers are used to classify and isolate stem cells and to monitor their differentiation state by antibody-based techniques. The expression of certain cell surface antigens is evidence for the cell's potency. However, because stem cells are heterogeneous in morphology, phenotype, and function, they need to be classified into subpopulations characterised by multiple sets of molecular biomarkers [17].

Human ESCs (hESCs) have flat compact colony morphology. Their growth depends on FGF and TGFb signalling. In 2007, the International Stem Cell Initiative characterised 59 hESCs lines from 17 laboratories worldwide [18]. Although the lines were not identical, presented various genotypes and were derived and maintained using different techniques, it was observed that they all exhibited similar expression patterns for several markers of human embryonic stem cells (Table 1).

Human iPSCs (hiPSCs) share all key features with human ESCs (hESCs), however there are some concerns about their molecular and functional equivalence discussed later in this article.

Adult tissue-derived stem cells (ASCs), such as mesenchymal stem cells (MSCs usually derived from bone marrow or fat but also many other tissues [20]) are among the most studied and some are currently being used in the clinic. In 2006, the International Society for Cellular Therapy (ISCT) produced a position statement suggesting the minimum criteria required to define MSCs [21]. They must be plastic adherent, differentiate into osteoblasts, adipocytes, and chondroblasts in vitro and present appropriate cell surface antigens expression (Table 2). 
A particular challenge for the field has been the absence of any specific marker to define MSCs, although a large number of different determinants have been associated with them but not exclusively. In vitro characterisation of MSC profiles of surface molecule expression is complicated by the fact that the culture conditions may introduce experimental artefacts. It has been proposed that certain natively expressed surface markers are modified following explantation, while new markers may be acquired [22].

\section{Problems with different stem cell sources}

Although there are various stem cell sources available, there are also many controversies and doubts as to their application in regenerative medicine. For example, embryonic stem cells (ESCs) have been shown to increase the risk for tumour formation [23]. In addition, their culture is a demanding task, which requires continuous maintenance of the undifferentiated state for long periods of time. Their stability during extended passaging cannot be guaranteed, and recent cytogenetic studies have shown karyotypic aberrations [24]. Because ESCs are genetically different from the recipient's cells they have the potential to generate immunological response and rejection, thereby creating the need for immunosuppressive therapy. Finally, there are many ethical issues and concerns surrounding their procurement from embryos. Thus, there are only about 300 NIH registry-approved human embryonic stem cell lines, which represents only $0.00001 \%$ of the world's human genetic diversity ( 7 billion people). New methods of deriving ESCs are investigated, among them parthenogenetic ESCs [25-27] or removal of a single blastomere from a morula stage embryo (embryo biopsy) [28, 29], but still more research is needed to consider them as safe and effective protocols.

Foetal stem cells are less ethically contentious than ESCs and their differentiation potential appears greater than adult stem cells [30]. They can be harvested from foetal blood and liver without harming the foetus. Some research show that they do not elicit alloreactive lymphocyte proliferation [31].

Adult tissue-derived stem cells usually are less tumourogenic than their embryonic counterparts and provide an autologous source of cells eliminating concerns regarding rejection and disease transmission [32]. However, their donordependency, limited expansion potential and a highly restricted differentiation capacity are serious disadvantages [33]. When considering ASCs as a source of stem cells for regenerative therapies it must be noted that harvesting them from tissues may present some technical problems (e.g. bone marrow stem cells - painful procedure, risk of infection). To acquire an appropriate amount of MSCs, to culture them maintaining their pluripotent characteristics and then to transplant them within tissues in vivo is a challenge, because the number and quality of MSCs derived depends on the type of tissue used as a source, but also on age and sex of the donor [34-36].

With iPSCs there are also many well-known technical problems that remain unresolved. Reprogramming adult cells to the embryonic stage is time consuming and requires special conditions and procedures that are not yet well established. The risk of acquiring somatic mutations and chromosomal rearrangements during the induction of pluripotency and reports of genetic and epigenetic variation between iPSC lines that were derived using identical methods are difficult issues to address [37]. The question of whether iPSCs are genuine copies of the cells that exist in vivo arises. Recent research shows that iPSCs may be significantly different from the cells found in tissues because they retain residual DNA methylation patterns typical of parental somatic cells $[38,39]$. However, there are also more and more reports on effective methods of deriving footprint-free iPSCs [40-42].

\section{Clinical application - the eye}

\section{Limbal stem cells deficiency}

The cornea is a complex, multi-layered, multifunctional, transparent structure in the anterior segment of an eye that provides photo protection, refraction, and transparency and helps protect internal ocular structures [43]. It is composed of various layers, the outermost of which is the epithelium. Its stem cells are found only in the palisades of Vogt located on the periphery of the cornea, in the region known as the limbus. Thus, they are called limbal stem cells (LSCs). The limbal epithelium consists of three cell types: stem cells, transient amplifying cells (TACs) that migrate to the central cornea devoid of stem cells to regenerate it, and differentiated cells. Isolating a pure population of stem cells from the palisades of Vogt is difficult [44].

Although LSCs have been the subject of research for many decades, no definitive marker has been identified so far [45]. They may be characterised as small active primitive cells that are p63, ABCG2, and integrin $\alpha 9$ positive and nestin, E-cadherin, connexin 43, involucrin, K3, and K12 negative, while they have a relatively higher expression of integrin $\beta 1$, EGFR, K19, and enolase- $\alpha$ [46-48]. However, there are researchers who point out that the use of some of these markers makes it impossible to isolate and characterise live LSCs because the cells have to be permeabilised, which results in loss of cell viability. Thus, the use of RHAMM/HMMR as a negative LSCs marker for successful clinical isolation has been suggested [49]. Many other markers of LSCs are still being investigated.

If the limbus is damaged, for example as a result of a chemical burn, limbal stem cells deficiency occurs (LSCD) and the cornea loses its transparency which results in blindness. If the LSCD is unilateral the autologous transplantation of limbal tissue from the uninjured 
eye may be performed [50]. Nevertheless, the procedure carries a risk of inducing LSCD in the donor eye due to the need of large limbal biopsy, necessitating the ex vivo expansion of LSCs as an alternative [51, 52]. It was shown that upon transplantation cultured autologous LSCs permanently restored corneal transparency of patients with ocular burns [53].

New sources of LSCs and the ways of their ex vivo expansion are constantly being investigated [54]. Hayashi et al. generated corneal epithelial cells from human iPSCs by method called stromal cell-derived inducing activity (SDIA) [55]. Ouyang et al. using exogenous expression of PAX6 reprogrammed epidermal cells into LSC-like cells that were able to repair damaged corneal surface on rabbit corneal injury model [56]. They did it with a new feeder-cell-free LSCs expansion protocol. Reports from Mikhailova et al. presented direct differentiation method for iPSs toward corneal epithelial progenitor cells capable of terminal differentiation to mature corneal epithelial-like cells using 2 small-molecule inhibitors in combination with basic fibroblast growth factor (bFGF) in serum-free and feeder-free conditions [57]. Another protocol proposed by Zhao included the use of amniotic membranes as a niche for LSCs ex vivo proliferation [58]. Autologous oral mucosal epithelium graft with amniotic membrane transplantation was also proved as a viable alternative to restore corneal surfaces suffering from total limbal stem cell deficiency [59].

\section{Glaucoma}

Glaucoma is a neuropathy of the optic nerve and one of the major causes of blindness in the world population. Although its aetiology is multifactoral and not yet fully understood, it is known that the visual impairment is a result of the damage to the retinal ganglion cells (RGCs). These are cells located in the innermost layers of the retina and their axons form optic nerve.

Through tissue-engineering techniques various types of stem cells have been found to be a potential source of RGCs for optic nerve regeneration. Stepwise, documented differentiation of RGCs through all of the major stages of retinogenesis is crucial for determining whether cells derived in vitro from ESCs [60], ASCs, or iPSCs are truly RGCs. Appropriate physiological activity should be proven as well.

Initially, RGC differentiation is triggered by fibroblast growth factors FGF3 and FGF8 released by organising centres in the optic stalk and neural retina [61]. First the axon's formation is initiated, and then dendrites start to occur [62]. Commonly identified markers for RGCs are Brn3, Islet1, and Pax6, but they are not specific for RGCs only [63]. Thus, identifying RGCs especially derived from other sources than the retina itself requires also checking their morphological and physiological features. Morphologically RGCs possess a very elongated axon and a sig- nificant number of dendritic extensions. Several protocols have been reported for RGCs differentiation [64, 65].

A very interesting source of RGCs can be Müller stem cells derived from retina. They can be harvested from a donor post-mortem and expanded in vitro as showed Limb et al. by creating an immortalized Müller cell line (MIO-M1) [66]. Cells from this line have been later transplanted into a glaucomatous rat model and expressed both neuronal and glial cell markers [67]. In another rat model, RGC precursors from the donor neural retina have been transplanted onto the inner retinal surface of rats depleted of RGCs where they integrated into tissues and continued expressing RGC's markers in vivo [68]. These experiments demonstrated that Müller stem cells can become oriented toward the optic nerve in vivo and potentially restore RGC function in glaucomatous eyes.

Among other ASCs used in optic nerve regeneration are oligodendrocyte precursor cells (OPCs) and olfactory ensheathing cells (OECs). In glaucoma there is a loss of oligodendrocytes which may play a role in the pathogenesis and progression of the disease [69]. Oligodendrocyte precursor cells posses the ability to migrate, proliferate, and differentiate into mature oligodendrocytes and ensheath demyelinated axons in animal models [70]. By producing IGF-1 and GDNF they also enhance the survival of neurons in vitro and in vivo [71, 72]. OECs transplanted into the host eye not only secrete neurotrophic factors stimulating the survival of RGCs but also migrate and integrate into the optic nerve and promote growth of RGCs [73-75].

Another research shows that ESCs injected into epiretinal space of mouse eye also efficiently integrate into the RGC layer and INL and they exhibit neuroprotective features over existing neurons [76].

When it comes to iPSCs they were shown to differentiate into RGC-like cells by introducing the transcription factors Dkk1 + Noggin (DN) + DAPT and overexpression of Math5 [77]. However, they were not able to be integrated into the normal retina after transplantation.

Retinal ganglion cell depletion is very often associated with increased intraocular pressure. Thus many researchers focus on regenerating trabecular meshwork (TM) which is a structure responsible for regulating the outflow of aqueous fluid from the anterior chamber of the eye and controlling intraocular pressure.

Du et al. extracted stem cells from trabecular meshwork and proved their capability of differentiating into cells expressing TM markers and exhibiting phagocytic function [78]. The cells were then transplanted into aqueous fluid and migrated to trabecular meshwork renewing TM cells population within it [79]. Also Tay et al. successfully propagated in vitro MSC cells derived from TM [80]. Manuguerra-Gagne et al. showed that bone marrow-derived mouse MSC mediate regeneration of damaged TM in vivo, apparently through production of paracrine factors [81]. Abu-hassan et al. differentiated human iPSCs into TM-like cells, 
that upon transplantation restored partially IOP homeostasis in an ex vivo human TM cell loss model, by introduction of TM cell-derived extra cellular matrix (ECM) and TM cell-derived conditioned media in culture [82]. Ding et al. also derived TM-like cells by coculturing of mouse iPSCs with primary human TM [83].

\section{Age-related macular degeneration}

Age-related macular degeneration (AMD) is a major cause of blindness in older population. It is a pathology which leads to retinal pigmented epithelium cells (RPE) atrophy and loss of photoreceptors in macular region of the eye. Advanced AMD, including neovascular (wet) type and geographic atrophy (dry type), is associated with substantial, progressive visual impairment. Current treatment for wet AMD incorporates intra ocular injections of anti-VEGF agents, occasionally combined with other methods. There is no efficient treatment for geographic atrophy known. For both types of AMD regenerative medicine could contribute successful therapeutic methods.

It has been demonstrated that healthy autologous RPE transplanted to macular region (using different techniques) in an early stage of AMD could partially restore vision [84-88]. However, because of the major drawbacks of these methods, such as high rate of complications and difficulties in collecting the number of RPE large enough to adequately repopulate macula, they did not contribute a satisfactory therapeutic option so far.

Several defined protocols were conducted to generate mature RPE cells from ESCs. Kawasaki et al. used coculture of ESCs with PA6 stromal cells acquiring cells expressing markers of mature RPE: ZO-1, RPE65, CRALBP, and MerTK [89]. The protocol resembled the natural generation of RPE cells and avoided the potential contaminations from recombinant proteins or small molecules which were used in other protocols but was characterized by low efficiency.

Other researchers investigated deriving RPE cells using mouse embryonic fibroblast cells as the feeder layer for hESCs and hiPSCs but it was shown that animal-derived components may cause unwanted immunogenicity of the generated cells because they carry factors such as sialic acid or Neu5Gc $[90,91]$. As an alternative Vaajasaari et al. and Zhang et al. proposed xeno-free protocols [92, 93].

RPE cells obtained through protocols presented by Meyer et al. [94] and Park et al. [95] were later tested for treatment of AMD and Stargard's disease [96]. Also Schwartz [97] et al. is investigating safety and tolerability of subretinal injection of hESC RPE cell suspensions.

\section{Growing an eye}

Most recent researches show promising advances in applying tissue engineering methods in attempts to grow a whole organ in vitro from stem cells. Hayashi et al. pre- sented a method of creating SEAM (self-formed ectodermal autonomous multi-zone) of ocular cells which mimics whole-eye development [98]. Human iPS cells were cultivated in differentiation medium in which they spontaneously and progressively formed a primordium comprising four identifiable concentric zones. Cell location within different zones was indicative of lineage, spanning the ocular surface ectoderm, lens, neuro-retina, and retinal pigment epithelium.

\section{Conclusions}

Stem cells provide a variety of potential cures for many diseases as it can be illustrated on the example of an eye. There are many sources they can be derived from and currently different methods of cultivating and using those cells in regenerative medicine are being investigated. Stem cells contribute a promising treatment in the future.

The authors declare no conflict of interest.

\section{References}

1. Trounson A, McDonald C (2015): Stem cell therapies in clinical trials: Progress and challenges. Cell Stem Cell 17: 11-22.

2. Evans MJ, Kaufman MH (1981): Establishment in culture of pluripotential cells from mouse embryos. Nature 292: 154156.

3. Lodi D, Iannitti T, Palmieri B (2011): Stem cells in clinical practice: applications and warnings. J Exp Clin Cancer Res 30: 9.

4. O'Donoghue K, Fisk NM (2004): Fetal stem cells. Best Pract Res Clin Obstet Gynaecol 18: 853-875.

5. Schwab M (2009): Encyclopedia of cancer. Springer-Verlag, Berlin, Heidelberg; 3511.

6. Skipper M, Weiss U, Gray N (2010). Plasticity. Nature 465: 703.

7. Schwab M (2009): Encyclopedia of cancer. Springer-Verlag, Berlin, Heidelberg; 3028-3029.

8. Takahashi K, Yamanaka S (2006): Induction of pluripotent stem cells from mouse embryonic and adult fibroblast cultures by defined factors. Cell 126: 663-676.

9. Smith A (2006): A glossary for stem-cell biology. Nature 441: 1060.

10. Wobus AM, Boheler KR (2005): Embryonic stem cells: prospects for developmental biology and cell therapy. Physiol Rev 85: 635-678.

11. Rossant J (2011): Stem cells from the mammalian blastocyst. Stem Cells 19: 477-482.

12. Kolios G, Moodley Y (2013): Introduction to Stem Cells and Regenerative Medicine. Respiration 85: 3-10.

13. Zhou H, Wu S, Joo JY, et al. (2009): Generation of induced pluripotent stem cells using recombinant proteins. Cell Stem Cell 8: 381-384.

14. Warren L, Manos PD, Ahfeldt T, et al. (2010): Highly efficient reprogramming to pluripotency and directed differentiation of human cells with synthetic modified mRNA. Cell Stem Cell 5: 618-630. 
15. Miyoshi N, Ishii H, Nagano H, et al. (2011): Reprogramming of mouse and human cells to pluripotency using mature microRNAs. Cell Stem Cell 3: 633-638.

16. Abad M, Mosteiro L, Pantoja C, et al. (2013): Reprogramming in vivo produces teratomas and iPS cells with totipotency features. Nature 502: 340-345.

17. Nagano K, Yoshida Y, Isobe T, et al. (2008): Cell surface biomarkers of embryonic stem cells. Proteomics 8: 4025-4035.

18. Adewumi O, Aflatoonian B, Ahrlund-Richter L, et al. (2007): Characterization of human embryonic stem cell lines by the International Stem Cell Initiative. International Stem Cell Initiative. Nat Biotechnol 25: 803-816.

19. Yu J, Thomson JA (2014): Induced pluripotent stem celss. In: Principles of Tissue Engineering. Lanza R, Langer R, Vacanti JP (eds.). Elsevier Inc; 581-594.

20. Dabrowski FA, Burdzinska A, Kulesza A, et al. (2017): Mesenchymal stem cells from human amniotic membrane and umbilical cord can diminish immunological response in an in vitro allograft model. Gynecol Obstet Invest 82: 267-275.

21. Dominici M, Le Blanc K, Mueller I, et al (2006): Minimal criteria for defining multipotent mesenchymal stromal cells. The International Society for Cellular Therapy position statement. Cytotherapy 8: 315-317.

22. Murray IR, West CC, Hardy WR, et al. (2014): Natural history of mesenchymal stem cells, from vessel walls to culture vessels. Cell Mol Life Sci CMLS 71: 1353-1374.

23. Karussis D, Kassis I, Kurkalli BG, Slavin S (2008): Immunomodulation and neuroprotection with mesenchymal bone marrow stem cells (MSCs): a proposed treatment for multiple sclerosis and other neuroimmunological/ neurodegenerative diseases. J Neurol Sci 265 (1-2): 131-135.

24. Baker DE, Harrison NJ, Maltby E, et al. (2007): Adaptation to culture of human embryonic stem cells and oncogenesis in vivo. Nat Biotechnol 25: 207-215.

25. Mai Q, Yu Y, Li T, et al. (2007): Derivation of human embryonic stem cell lines from parthenogenetic blastocysts. Cell Res 17: 1008-1019.

26. Revazova ES, Turovets NA, Kochetkova OD, et al. (2008): HLA homozygous stem cell lines derived from human parthenogenetic blastocysts. Cloning Stem Cells 10: 11-24.

27. Brevini TA, Gandolfi F (2008): Parthenotes as a source of embryonic stem cells. Cell Prolif 41 (Suppl.1): 20-30

28. Klimanskaya I, Chung Y, Becker S, et al. (2007): Derivation of human embryonic stem cells from single blastomeres. Nat Protoc 2: 1963-1972.

29. Chung Y, Klimanskaya I, Becker S, et al. (2008): Human embryonic stem cell lines generated without embryo destruction. Cell Stem Cell 2: 113e7.

30. O'Donoghue K, Fisk NM (2004): Fetal stem cells, Best Pract Res Clin Obstet Gynaecol 18: 853-887.

31. Götherström C, Ringdén O, Tammik C, et al. (2004): Immunologic properties of human fetal mesenchymal stem cells. Am J Obstet Gynecol 190: 239-245.

32. Khan W, Hardingham T (2012): Mesenchymal Stem Cells, Sources of Cells and Differentiation Potential. J Stem Cells 7: 75-85.

33. Wilson KD, Wu JC (2015): Induced pluripotent stem cells. JAMA 313: 1613-1614.

34. Stolzing A, Jones E, McGonagle D, Scutta A (2008): Age-related changes in human bone marrow-derived mesenchymal stem cells: Consequences for cell therapies. Mech Ageing Dev 129: 163-173.
35. Lohmann M, Walenda G, Wagner W, et al. (2012): Donor age of human platelet lysate affects proliferation and differentiation of mesenchymal stem cells. PloS One 7: 1-11

36. Rojewski MT, Weber BM, Schrezenmeier H (2008): Phenotypic characterization of mesenchymal stem cells from various tissues. Transfus Med Hemother 35: 168-184.

37. Wilson KD, Wu JC (2015): Induced pluripotent stem cells. JAMA 313: 1613-1614.

38. Kim K, Doi A, Wen B, et al. (2010): Epigenetic memory in induced pluripotent stem cells. Nature 467: 285-290.

39. Ma H, Morey R, O'Neil RC, et al. (2014). Abnormalities in human pluripotent cells due to reprogramming mechanisms. Nature 511: 177.

40. Choi HY, Lee TJ, Yang GM, et al. (2016): Efficient mRNA delivery with graphene oxide-polyethylenimine for generation of footprint-free human induced pluripotent stem cells. J Control Release 235: 222-235.

41. Boreström C, Simonsson S, Enochson L, et al. (2014): Footprint-free human induced pluripotent stem cells from articular cartilage with redifferentiation capacity: a first step toward a clinical-grade cell source. Stem Cells Transl Med 3: 433447.

42. Mormone E, D'Sousa S, Alexeeva V, et al. (2014): "Footprint-free" human induced pluripotent stem cell-derived astrocytes for in vivo cell-based therapy. Stem Cells Dev 23: 2626-2636.

43. Thomas PB, Liu YH, Zhuang FF, et al. (2007): Identification of Notch-1 expression in the limbal basal epithelium. Mol Vis 13: 337-344.

44. Lehrer MS, Sun TT, Lavker RM (1998): Strategies of epithelial repair: modulation of stem cell and transit amplifying cell proliferation. J Cell Sci 111: 2867-2875.

45. Ebrahimi M, Taghi-Abadi E, Baharvand H (2009): Limbal stem cells in review. J Ophthalmic Vis Res 4: 40-58.

46. Chen Z, de Paiva CS, Luo L, et al. (2004): Characterization of putative stem cell phenotype in human limbal epithelia. Stem Cells 22: 355-366.

47. Schlotzer-Schrehardt U, Kruse FE (2005): Identification and characterization of limbal stem cells. Exp Eye Res 81: 247264.

48. Eichner R, Bonitz P, Sun TT (1984): Classification of epidermal keratins according to their immunoreactivity, isoelectric point, and mode of expression. J Cell Biol 98: 1388-1396.

49. Ahmad S, Kolli S, Li DQ, et al. (2008): A putative role for RHAMM/HMMR as a negative marker of stem cell-containing population of human limbal epithelial cells. Stem Cells 26: 1609-1619.

50. Kenyon KR, Tseng S (1989): Limbal autograft transplantation for ocular surface disorders. Ophthalmology 96: 709-723.

51. Vazirani J, Basu S, Kenia H, et al. (2014): Unilateral partial limbal stem cell deficiency: Contralateral versus ipsilateral autologous cultivated limbal epithelial transplantation. Am J Ophthalmol 157 (3): 584-590.

52. Pellegrini G, Ranno R, Stracuzzi G, et al. (1999): The control of epidermal stem cells (holoclones) in the treatment of massive full-thickness burns with autologous keratinocytes cultured on fibrin. Transplantation 68: 868-879.

53. Rama P, Bonini S, Lambiase A, et al. (2001): Autologous fibrin-cultured limbal stem cells permanently restore the corneal surface of patients with total limbal stem cell deficiency. Transplantation 72: 1478-1485. 
54. Woronkowicz M, Skopinski P (2010): Clinical applications of limbal epithelial stem cells. Centr Eur Jo Immunol 35: 179182.

55. Hayashi R, Ishikawa Y, Kageyama T, et al. (2012): Generation of corneal epithelial cells from induced pluripotent stem cells derived from human dermal fibroblast and corneal limbal epithelium. PloS One 7: 1-10.

56. Ouyang H, Xue Y, Lin Y, et al. (2014): WNT7A and PAX6 define corneal epithelium homeostasis and pathogenesis. $\mathrm{Na}-$ ture 511: 358-361.

57. Mikhailova A, Ilmarinen T, Uusitalo H, et al. (2014): Small-molecule induction promotes corneal epithelial cell differentiation from human inducedpluripotent stem cells. Stem Cell Reports 2: 219-231.

58. Zhao Y, Ma L (2015): Systematic review and meta-analysis on transplantation of ex vivo cultivated limbal epithelial stem cell on amniotic membrane in limbal stem cell deficiency. Cornea 34: 592-600.

59. Liu J, Sheha H, Fu Y, et al. (2011): Oral mucosal graft with amniotic membrane transplantation for total limbal stem cell deficiency. Am J Ophthalmol 152: 739-747.

60. Woronkowicz M, Skopinski P (2010): Embryonic stem cells and retinal regeneration. Centr Eur J Immunol 35: 267-272.

61. Martinez-Morales JR, Del Bene F, Nica G, et al. (2005): Differentiation of the vertebrate retina is coordinated by an FGF signaling center. Developmental Cell 8: 565-574.

62. Sun Y, Williams A, Waisbourd M, et al. (2015): Stem cell therapy for glaucoma: Science or snake oil? Survey of Ophthalmology 60: 93-105.

63. Cooke JA, Meyer JS (2015): Human pluripotent stem cell-derived retinal ganglion cells: applications for the study and treatment of optic neuropathies. Curr Ophthalmol Rep 3: 200-206.

64. Riazifar H, Jia Y, Chen J, et al. (2014): Chemically induced specification of retinal ganglion cells from human embryonic and induced pluripotent stem cells. Stem Cells Transl Med 3: 424-432.

65. Maekawa Y, Onishi A, Matsushita K, et al. (2016): Optimized culture system to induce neurite outgrowth from retinal ganglion cells in three-dimensional retinal aggregates differentiated from mouse and human embryonic stem cells. Curr Eye Res 41: 558-568.

66. Limb GA, Salt TE, Munro PM, et al. (2002): In vitro characterization of a spontaneously immortalized human Muller cell line (MIO-M1). Invest Ophthalmol Vis Sci 43: 864-869.

67. Bull ND, Limb GA, Martin KR (2008): Human Muller stem cell (MIOM1) transplantation in a rat model of glaucoma: survival, differentiation, and integration. Invest Ophthalmol Vis Sci 49: 3449-3456.

68. Singhal S, Bhatia B, Jayaram H, et al. (2012): Human Muller glia with stem cell characteristics differentiate into retinal ganglion cell (RGC) precursors in vitro and partially restore RGC function in vivo following transplantation. Stem Cells Transl Med 1: 188-199.

69. Son JL, Soto I, Oglesby E, et al. (2010): Glaucomatous optic nerve injury involves early astrocyte reactivity and late oligodendrocyte loss. Glia 58: 780-789.

70. Watanabe M, Toyama Y, Nishiyama A (2002): Differentiation of proliferated NG2-positive glial progenitor cells in a remyelinating lesion. J Neurosci Res 69: 826-836.

71. Wilkins A, Chandran S, Compston A (2001): A role for oligodendrocyte-derived IGF-1 in trophic support of cortical V neurons. Glia 36: 48-57.
72. Wilkins A, Majed H, Layfield R, et al. (2003): Oligodendrocytes promote neuronal survival and axonal length by distinct intracellular mechanisms: a novel role for oligodendrocyte-derived glial cell line-derived neurotrophic factor. J Neurosci 23: 4967-4974.

73. Li Y, Sauve Y, Li D, et al. (2003): Transplanted olfactory ensheathingcells promote regeneration of cut adult rat optic nerve axons. J Neurosci 23: 7783-7788.

74. Leaver SG, Harvey AR, Plant GW (2006): Adult olfactory ensheathing glia promote the long-distance growth of adult retinal ganglion cell neurites in vitro. Glia 53: 467-476.

75. Xie BB, Zhang XM, Hashimoto T, et al. (2014): Differentiation of retinal ganglion cells and photoreceptor precursors from mouse induced pluripotent stem cells carrying an Atoh7/ Math5 lineage reporter. PLoS One 9: e112175.

76. Hambright D, Park KY, Brooks M, et al. (2012): Long-term survival and differentiation of retinal neurons derived from human embryonic stem cell lines in un-immunosuppressed mouse retina. Molecular Vision 18: 920-936.

77. Chen M, Chen Q, Sun X, et al. (2010): Generation of retinal ganglionlike cells from reprogrammed mouse fibroblasts. Invest Ophthalmol Vis Sci 51: 5970-5978.

78. Du Y, Roh DS, Mann MM, et al. (2012): Multipotent stem cells from trabecular meshwork become phagocytic TM cells. Invest Ophthalmol Vis Sci 53: 1566-1575.

79. Du Y, Yun H, Yang E, et al. (2013): Stem cells from trabecular meshwork home to TM tissue in vivo. Invest Ophthalmol Vis Sci 54: 1450-1459.

80. Tay CY, Sathiyanathan P, Chu SW, et al. (2012): Identification and characterization of mesenchymal stem cells derived from the trabecular meshwork of the human eye. Stem Cells Dev 21: 1381-1390.

81. Manuguerra-Gagne R, Boulos PR, Ammar A, et al. (2013): Transplantation of mesenchymal stem cells promotes tissue regeneration in a glaucoma model through laser-induced paracrine factor secretion and progenitor cell recruitment. Stem Cells 31: 1136-1148.

82. Abu-Hassan DW, Li X, Ryan EI, et al. (2015): Induced pluripotent stem cells restore function in a human cell loss model of open-angle glaucoma. Stem Cells 33: 751-761.

83. Ding QJ, Zhu W, Cook AC, et al. (2014): Induction of trabecular meshwork cells from induced pluripotent stem cells. Invest Ophthalmol Vis Sci 55: 7065-7072.

84. Takeuchi K, Kachi S, Iwata E, et al. (2012): Visual function 5 years or more after macular translocation surgery for myopic choroidal neovascularisation and age-related macular degeneration. Eye (Lond) 26: 51-60.

85. Chen FK, Patel PJ, Uppal GS, et al. (2010): Long-term outcomes following full macular translocation surgery in neovascular age-related macular degeneration. Br J Ophthalmol 94: 1337-1743.

86. Falkner-Radler CI, Krebs I, Glittenberg C, et al. (2011): Human retinal pigment epithelium (RPE) transplantation: outcome after autologous RPE-choroid sheet and RPE cell-suspension in a randomized clinical study. Br J Ophthalmol 95: 370-375.

87. MacLaren RE, Uppal GS, Balaggan KS, et al. (2007): Autologous transplantation of the retinal pigment epithelium and choroid in the treatment of neovascular age-related macular degeneration. Ophthalmology 114: 561-570.

88. Binder S, Krebs I, Hilgers RD, et al. (2004): Outcome of transplantation of autologous retinal pigment epithelium in age-related macular degeneration: a prospective trial. Invest Ophthalmol Vis Sci 45: 4151-4160. 
89. Kawasaki H, Suemori H, Mizuseki K, et al. (2002): Generation of dopaminergic neurons and pigmented epithelia from primate ES cells by stromal cell-derived inducing activity. Proc Natl Acad Sci U S A 99: 1580-1585.

90. Martin MJ, Muotri A, Gage F, Varki A (2005): Human embryonic stem cells express an immunogenic nonhuman sialic acid. Nat Med 11: 228-232.

91. Sakamoto N, Tsuji K, Muul LM, et al. (2007): Bovine apolipoprotein B-100 is a dominant immunogen in therapeutic cell populations cultured in fetal calf serum in mice and humans. Blood 110: 501-508.

92. Vaajasaari H, Ilmarinen T, Juuti-Uusitalo K, et al. (2011): Toward the defined and xeno-free differentiation of functional human pluripotent stem cell-derived retinal pigment epithelial cells. Mol Vis 17: 558-575.

93. Zhang YS, Lu ZY, Yu Y, et al. (2012): Derivation, culture and retinal pigment epithelial differentiation of human embryonic stem cells using human fibroblast feeder cells. J Assist Reprod Genet 29: 735-744.

94. Meyer JS, Shearer RL, Capowski EE, et al. (2009): Modeling early retinal development with human embryonic and induced pluripotent stem cells. Proc Natl Acad Sci U S A 106: 1669816703.

95. Park UC, Cho MS, Park JH, et al. (2011): Subretinal transplantation of putative retinal pigment epithelial cells derived from human embryonic stem cells in rat retinal degeneration model. Clin Exp Reprod Med 38: 216-221.

96. Lu B, Malcuit C, Wang S, et al. (2009): Long-term safety and function of RPE from human embryonic stem cells in preclinical models of macular degeneration. Stem Cells 27: 2126-2135.

97. Schwartz SD, Regillo CD, Lam BL, et al. (2015): Human embryonic stem cell-derived retinal pigment epithelium in patients with age-related macular degeneration and Stargardt's macular dystrophy: follow-up of two open-label phase studies. Lancet 9967: 509-516.

98. Hayashi R, Ishikawa Y, Sasamoto Y, et al. (2016): Co-ordinated ocular development from human iPS cells and recovery of corneal function. Nature 531: 376-380. 